\title{
Hierarchy of equations of motion for nonlinear coherent excitations applied to magnetic vortices
}

\author{
F. G. Mertens*, H. J. Schnitzer*, and A. R. Bishop \\ Theoretical Division, Los Alamos National Laboratory, MS B262, Los \\ Alamos, New Mexico 87545 \\ *Physikalisches Institut, Universität Bayreuth, D-95440 Bayreuth, \\ Germany
}

\begin{abstract}
Starting from a travelling wave ansatz we show successively that the shape of a nonlinear excitation generally depends also on the $1^{\text {st }}, 2^{\text {nd }}, \ldots$ time derivative of the position $\vec{X}$ of the excitation. From the Hamilton equations we derive a hierarchy of equations of motion for $\vec{X}$. The type of the excitation determines on which levels the hierarchy can be truncated consistently: "Gyrotropic" excitations are governed by odd-order equations, non-gyrotropic ones by even-order equations. Examples for the latter case are kinks in 1-dimensional models and planar vortices of the 2-d anisotropic (easy-plane) Heisenberg model. The nonplanar vortices of this model are the simplest gyrotropic example. For this case we solve the Hamilton equations for a finite system with one vortex and free boundary conditions and calculate the parameters of the $3^{\text {rd }}$-order equation of motion. This equation yields trajectories which are a superposition of two cycloids with different frequencies, which is in full agreement with computer simulations of the full many-spin model. Finally we demonstrate that the additional effects from the $5^{\text {th }}$-order equation are negligible.
\end{abstract}

\section{Introduction}

Nonlinear coherent excitations, such as solitons or solitary waves, usually have some particle-like properties. E.g., the equation of motion of their 'center-of-mass' is New- 
tonian, at least in a first approximation. For the case of zero force the excitations can move at constant velocity due to their inertial mass. However, there are also other excitations, which do not behave like classical particles, i.e., Galilei's law is not valid. For spin systems, good examples are certain magnetic domains and non-planar vortices in two- or three-dimensional models. For this type of nonlinear collective excitations Thiele [1, 2] derived a $1^{\text {st }}$-order equation of motion for the collective variable $\vec{X}(t)$

$$
\dot{\vec{X}} \times \vec{G}=\vec{F},
$$

which we will refer to as the Thiele Eq. in the following. It is valid only for steadystate motion because it was derived from the Landau-Lifshitz equation for spins assuming a rigid shape of the excitations.

$\vec{F}$ is either an external force or the force due to interactions with other excitations. $\vec{V} \times \vec{G}$ is called a gyrocoupling force. It is formally equivalent to the Lorentz force. However $\vec{G}$ is not an external magnetic field but an intrinsic quantity, produced by the excitation itself and carried along with it. $\vec{G}$ is called the gyrocoupling vector, or for short gyrovector. We call excitations with $|\vec{G}|=G \neq 0$ gyrotropic. For 1-d spin models $\vec{G}$ is always zero. For the vortices of the 2-d anisotropic Heisenberg model

$$
\vec{G}=2 \pi q p \vec{e}_{z}
$$

as was first calculated by Huber [3]. Here $\vec{e}_{z}$ is the unit vector perpendicular to the $x y$-plane in which the magnetic ions are situated. $q= \pm 1, \pm 2, \ldots$ is the vorticity, $p$ is a second topological charge which is defined as the value of the spin component $S^{z}$ at the vortex center in the continuum limit.

The 2-d anisotropic Heisenberg model with $X Y$-symmetry is defined by the spin Hamiltonian

$$
H=-J \sum_{<m, n>}\left[S_{m}^{x} S_{n}^{x}+S_{m}^{y} S_{n}^{y}+(1-\delta) S_{m}^{z} S_{n}^{z}\right]
$$

with $0<\delta \leq 1$. Here $<m, n>$ labels the nearest-neighbor sites of a square lattice. We treat the spin $\vec{S}$ as a classical vector and set $S=J=1$.

Due to the anisotropy $\delta$ the spins prefer to be oriented in the $x y$-plane which is therefore often called easy plane. For strong anisotropy $\left(\delta_{c}<\delta \leq 1\right.$, with $\left.\delta_{c} \simeq 0.28\right)$ only planar vortices are stable $[4,5]$. In the static case their spins are lying completely in the plane, while dynamically small $S^{z}$-components develop. But at the vortex center $S^{z}$ is always zero, thus $p=0$ and $G=0$ due to (2). Therefore the Thiele Eq. is not valid here.

In contrast to this case, for weak anisotropy $\left(0<\delta<\delta_{c}\right)$ only non-planar vortices are stable [4. They exhibit a localized structure of the $S^{z}$-components around the vortex center, at which $S^{z}= \pm 1$. Although this structure changes due to motion, the value at the center remains the same (section 3). Thus $p= \pm 1$ determines to which side the out-of-plane structure of the vortex is oriented and is therefore be termed polarization. The Thiele Eq. is valid for steady-state motion when the vortex shape 
is rigid (in the moving frame). This includes the case of a constant rotation on a circle.

In 1994 Wysin et al. [6] dropped the rigid-shape assumption by allowing the vortex shape to depend on the velocity $\dot{\vec{X}}(t)$ and derived a generalized Thiele equation

$$
M \ddot{\vec{X}}+\dot{\vec{X}} \times \vec{G}=\vec{F}
$$

As the velocity dependent parts of the vortex structure decay like $1 / r$ with the distance $r$ from the vortex center [4], the mass was predicted to be

$$
M \simeq \frac{\pi}{4 \delta} \ln \left(L / a_{0}\right)
$$

where $L$ and $a_{0}$ are upper and lower cut-offs in the order of the system size and lattice constant, respectively. The same mass also appears in the kinetic energy $M \dot{X}^{2} / 2$ of a vortex [4].

According to Eq. (4) the trajectory $\vec{X}(t)$ of the vortex center is formally the same as that of an electric charge $e$ in a plane with a perpendicular magnetic field $\vec{B}$ and an in-plane electric force $\vec{F}$, namely a cycloid with frequency $\omega=G / M$, cf. the cyclotron frequency $e B /(M c)$, where $c$ is the speed of light. However, a test of this prediction by computer simulations turned out to be rather difficult: For the model (3) the use of an external force creates additional nonlinear excitations; e.g., an inplane magnetic field creates a double domain wall which connects the vortex with a boundary [7]. Therefore 2-vortex simulations were performed where each vortex is driven by the force between them [8, 9]. Large square systems with free boundaries were used and the trajectories were chosen such that the vortices stayed far away from each other and from the boundaries.

A 2-vortex theory was developed [9] which explains one very important qualitative feature of the simulations: There are four main scenarios in vortex dynamics, however cycloidal oscillations around a mean trajectory $\vec{X}^{0}(t)$ can be observed only for two of them, namely where either a vortex and an antivortex (i.e. $q_{1}=-q_{2}$ ) rotate around each other, or where two vortices with equal vorticity perform a parallel translation with constant average speed. There are no oscillations for the other 2 scenarios of 'vortex-vortex rotation' and 'vortex-antivortex translation'.

However, the frequency $\omega$ of the observed cycloidal oscillations yielded a mass" $M=G / \omega=2 \pi / \omega$ which was much larger than predicted by Eq. (5), see Table Ib of Ref. 9]. Later a second severe discrepancy was discovered after having improved the simulations for the 2-vortex rotation [10]: Better initial conditions were used and instead of a square a circular system was used, with symmetric initial positions. Here for each vortex the mean trajectory $\vec{X}^{o}(t)$ is a circle; the spectrum of the oscillations can be measured much more accurately and shows very clearly two frequencies $\omega_{1,2}$

\footnotetext{
${ }^{1}$ More precisely, for a 2-vortex system an effective mass tensor was defined, its eigenvalues were both much larger than predicted.
} 
with about the same strength, instead of the one frequency $\omega=G / M$ predicted by Eq. (雨).

Because of the above two discrepancies between theory and simulations we now derive a new theory which amounts to a hierarchy of equations of motion for a nonlinear coherent excitation in a system with an arbitrary Hamiltonian (section 2). The derivation is completely general, but for simplicity we consider a Hamiltonian which is a functional of only one field and its canonical momentum; this is the case of our model (3) in the continuum limit.

The level $n$ in this hierarchy is defined as the order $n$ of the highest time derivative which appears in the equation of motion. A classification of the excitations determines on which levels the hierarchy can be truncated consistently: The dynamics of gyrotropic excitations is described by odd-order equations, the simplest example is the non-planar vortex of model (3). Non-gyrotropic excitations (e.g. kinks in 1-d models, planar vortices in model (3)) are governed by even-order equations of motion.

In order to obtain the above classification one must know at least the order of magnitude of the parameters in the equations of motion. For the calculation of the parameters it is necessary to solve the Hamilton equations for a system with one excitation, choosing appropriate boundary conditions. We do this for our model (3) in section 3 using a finite system with free boundaries.

After the calculation of the parameters the equation of motion can be solved. For the non-planar vortices we solve a $3^{\text {rd }}$-order equation, which in fact yields the observed frequencies $\omega_{1,2}$ (the trivial solution $\omega=0$ yields the mean trajectory $\vec{X}^{o}(t)$ ), section 4. We compare with new simulations where a single vortex on a circle is driven by its image vortex (section 5). We only note that the 2 -vortex simulations yield a similar spectrum but cannot be compared quantitatively with this 1-vortex theory. We also show that the $5^{\text {th }}$-order equation, which appears on the next consistent level of the hierarchy, predicts two additional frequencies which can also be observed, but they are very weak. Therefore all levels higher than three can safely be neglected in this case.

In section 6 we discuss our results and in the appendix we describe the numerical part of this work in more detail.

\section{Hierarchy of equations of motion}

We consider an arbitrary classical Hamiltonian which is a functional of a field $\phi(\vec{r}, t)$ and its canonical momentum $m(\vec{r}, t)$. We introduce a collective variable $\vec{X}(t)$ for the position of a nonlinear coherent excitation. We make a travelling wave ansatz $\phi(\vec{r}, t)=\phi(\vec{r}-\vec{X}(t)), m(\vec{r}, t)=m(\vec{r}-\vec{X}(t))$ where, strictly speaking, the functions on the r.h.s. should bear an index, which is omitted here for simplicity. We insert

$$
\dot{\phi}=\frac{\partial \phi}{\partial X_{j}} \dot{X}_{j} \quad, \quad \dot{m}=\frac{\partial m}{\partial X_{j}} \dot{X}_{j}
$$


where a summation over $j=1,2,3$ is implied, into the Hamilton equations

$$
\dot{\phi}=\frac{\delta H}{\delta m} \quad, \quad \dot{m}=-\frac{\delta H}{\delta \phi} .
$$

Since the r.h.s. of these equations generally contain $m$ and $\phi$ it is clear that $m$ and $\phi$ depend not only on $\vec{X}$ but also on $\dot{\vec{X}}$. Thus the shape of the collective excitations generally depends on the velocity and we take this into account by the improved ansatz $\phi(\vec{r}, t)=\phi(\vec{r}-\vec{X}, \dot{\vec{X}}), m(\vec{r}, t)=m(\vec{r}-\vec{X}, \dot{\vec{X}})$. However, if we now insert $\dot{\phi}$ and $\dot{m}$ into the eqs. (17) we see an additional dependence on $\ddot{\vec{X}}$, and so on. Obviously we must truncate somewhere. We will see below that a truncation at the derivative $\vec{X}^{(n-1)}$ yields an $n^{\text {th }}$-order equation of motion. For gyrotropic excitations only oddorder equations will turn out to be consistent (section 4 ). As an example we derive here the $3^{\text {rd }}$-order equation by the ansatz

$$
\begin{aligned}
\phi(\vec{r}, t) & =\phi(\vec{r}-\vec{X}(t), \dot{\vec{X}}(t), \ddot{\vec{X}}(t)) \\
m(\vec{r}, t) & =m(\vec{r}-\vec{X}(t), \dot{\vec{X}}(t), \ddot{\vec{X}}(t)) .
\end{aligned}
$$

It is unusual that a collective excitation should depend on the acceleration, but it will be seen later that this dependence yields one of the dominant terms in the equation of motion. This equation is now easily derived by using the technique of Wysin et al. [6]. Our ansatz is inserted into the Hamilton equations (7) and yields

$$
\begin{aligned}
\frac{\partial \phi}{\partial X_{j}} \dot{X}_{\mathrm{J}}+\frac{\partial \phi}{\partial \dot{X}_{j}} \ddot{X}_{j}+\frac{\partial \phi}{\partial \ddot{X}_{j}} \dddot{X}_{j}= & \frac{\delta H}{\delta m} \\
\frac{\partial m}{\partial X_{j}} \dot{X}_{\mathrm{J}}+\frac{\partial m}{\partial \dot{X}_{j}} \ddot{X}_{j}+\frac{\partial m}{\partial \ddot{X}_{j}} \dddot{X}_{j}= & -\frac{\delta H}{\delta \phi}
\end{aligned}
$$

where $H$ depends on $\vec{X}, \dot{\vec{X}}$ and $\ddot{\vec{X}}$ via $\phi$ and $m$. Multiplying these equations by $\frac{\partial m}{\partial X_{i}}$ and $\frac{\partial \phi}{\partial X_{i}}$, resp., subtracting the equations from each other, and integrating over $\vec{r}$ we obtain

$$
\mathbf{A} \ddot{\vec{X}}+\mathbf{M} \ddot{\vec{X}}+\mathbf{G} \dot{\vec{X}}=\vec{F}
$$

Here

$$
F_{i}=-\int d^{3} r \frac{\partial \mathcal{H}}{\partial X_{i}}
$$

with the Hamiltonian density $\mathcal{H}$ is the static force, it is either an external force or the force due to the interactions with other nonlinear excitations.

$$
G_{i j}=\int d^{3} r\left\{\frac{\partial \phi}{\partial X_{i}} \frac{\partial m}{\partial X_{j}}-\frac{\partial \phi}{\partial X_{j}} \frac{\partial m}{\partial X_{i}}\right\}
$$

is equivalent to the gyrocoupling tensor of Thiele [1], 2]. Because of the antisymmetry of $\mathbf{G}$ the term $\mathbf{G} \dot{\vec{X}}$ can be written as $-\vec{G} \times \dot{\vec{X}}$. The gyrovector $\vec{G}$ is orthogonal to 
the plane defined by $\vec{X}$ and $\dot{\vec{X}}$ and has already been discussed in the introduction. The terms G $\dot{\vec{X}}$ and $\vec{F}$ constitute the Thiele Eq. valid only for steady-state motion. We note that the above method is much shorter than the original one by Thiele, who started directly from the Landau-Lifshitz Eq. (54).

$$
M_{i j}=\int d^{3} r\left\{\frac{\partial \phi}{\partial X_{i}} \frac{\partial m}{\partial \dot{X}_{j}}-\frac{\partial \phi}{\partial \dot{X}_{j}} \frac{\partial m}{\partial X_{i}}\right\}
$$

is referred to as the mass tensor, and

$$
A_{i j}=\int d^{3} r\left\{\frac{\partial \phi}{\partial X_{i}} \frac{\partial m}{\partial \ddot{X}_{j}}-\frac{\partial \phi}{\partial \ddot{X}_{j}} \frac{\partial m}{\partial X_{i}}\right\}
$$

as the $3^{\text {rd }}$-order gyrotensor. In order to see which properties they have it is necessary to calculate the functions $\phi$ and $m$ in the ansatz (8) which describe the shape of the excitations. We do this for our model (3).

\section{Hamilton equations for a finite system with one vortex and free boundaries}

We consider the 2-d anisotropic Heisenberg model (3) and introduce

$$
\begin{aligned}
\phi(\vec{r}, t) & =\tan ^{-1} \frac{S^{y}(\vec{r}, t)}{S^{x}(\vec{r}, t)} \\
m(\vec{r}, t) & =S^{z}(\vec{r}, t),
\end{aligned}
$$

where the site index at the spin components has been replaced by the variable $\vec{r}=$ $\left(x_{1}, x_{2}\right)$. The Hamiltonian is [4]

$$
H=\frac{1}{2} \int d^{2} r\left\{\left(1-m^{2}\right)(\nabla \phi)^{2}+\delta\left[4 m^{2}-(\nabla m)^{2}\right]+\frac{(\nabla m)^{2}}{1-m^{2}}\right\}
$$

and the Hamilton equations read

$$
\begin{aligned}
\frac{\partial \phi}{\partial t} & =m\left[4 \delta-(\nabla \phi)^{2}\right]-\left(\frac{1}{1-m^{2}}-\delta\right) \Delta m-\frac{m(\nabla m)^{2}}{\left(1-m^{2}\right)^{2}} \\
\frac{\partial m}{\partial t} & =\left(1-m^{2}\right) \Delta \phi-2 m \nabla m \nabla \phi .
\end{aligned}
$$

For $0<\delta<\delta_{c}$ (with $\delta_{c} \approx 0.28$ for a square lattice) only non-planar vortices are stable and they have the following static structure [4

$$
\phi_{0}=q \tan ^{-1} \frac{x_{2}^{\prime}}{x_{1}^{\prime}}+\varphi_{0}
$$




$$
\begin{aligned}
& m_{0}=p a_{2} \sqrt{\frac{r_{V}}{r^{\prime}}} e^{-r^{\prime} / r_{V}} \quad \text { for } r^{\prime} \gg r_{V} \\
& m_{0}=p\left[1-a_{1}^{2}\left(\frac{r^{\prime}}{r_{V}}\right)^{2}\right] \quad \text { for } r^{\prime} \ll r_{V}
\end{aligned}
$$

with

$$
\vec{r}^{\prime}=\vec{r}-\vec{X}
$$

The polarization is $p= \pm 1$ and only vorticities $q= \pm 1$ are considered. The constants $a_{1}$ and $a_{2}$ can be determined by matching the two solutions (19) and (20) at some intermediate distance, e. g. at $r \approx r_{V}$. The characteristic length

$$
r_{V}=\frac{1}{2} \sqrt{\frac{1-\delta}{\delta}}
$$

is interpreted as the radius of the vortex core. The constant $\varphi_{0}$ in (18) stays arbitrary. From the static structure one obtains for (12)

$$
G_{i j}=G \epsilon_{i j}
$$

with $G=2 \pi q p . \epsilon_{i j}$ is the 2 -d completely antisymmetric tensor.

For a slowly moving vortex we expand in a perturbation series

$$
\begin{aligned}
\phi & =\phi_{0}\left(\vec{r}^{\prime}\right)+\phi_{1}\left(\vec{r}^{\prime}, \dot{\vec{X}}\right)+\phi_{2}\left(\vec{r}^{\prime}, \ddot{\vec{X}}\right) \\
m & =m_{0}\left(\vec{r}^{\prime}\right)+m_{1}\left(\vec{r}^{\prime}, \dot{\vec{X}}\right)+m_{2}\left(\vec{r}^{\prime}, \ddot{\vec{X}}\right)
\end{aligned}
$$

and assume that the first and second order terms depend linearly on $\dot{X}_{j}$ and $\ddot{X}_{j}$, resp. Slow motion means here that the velocity is much smaller than the spin-wave velocity which is $2 \sqrt{\delta}$ in our dimensionless units [7].

In the next section it will be seen that the main contributions to the integrals for $\mathbf{M}$ and $\mathbf{A}$ stem from the region far away from the vortex center, i.e. from $r^{\prime} \gg r_{V}$. Particularly the dependence on the system size naturally comes entirely from this region. Therefore it is sufficient to solve the Hamilton equations only for this region. We assume that here only the terms $4 \delta m$ and $\Delta \phi$ on the r.h.s. of (17a) and (17b), resp., are important for the dynamic parts of $\phi$ and $m$. This assumption can be justified a posteriori.

In $O\left(\dot{X}_{j}\right)$ we then obtain for $r^{\prime} \gg r_{V}$

$$
\frac{\partial \phi_{0}}{\partial X_{j}} \dot{X}_{j}=4 \delta m_{1} \quad, \quad \frac{\partial m_{0}}{\partial X_{j}} \dot{X}_{j}=\Delta \phi_{1}
$$

The first equation gives

$$
m_{1}=\frac{q}{4 \delta\left(r^{\prime}\right)^{2}}\left(x_{2}^{\prime} \dot{X}_{1}-x_{1}^{\prime} \dot{X}_{2}\right)=\frac{q \sin \varphi^{\prime}}{4 \delta r^{\prime}}|\dot{\vec{X}}|,
$$


where $\varphi^{\prime}$ is the angle between $\vec{r}^{\prime}$ and $\dot{\vec{X}}$. For the second equation in (25) the inhomogenity vanishes at large distances. We therefore have the solution

$$
\phi_{1}=p c x_{j}^{\prime} \dot{X}_{j}
$$

for large $r^{\prime}$ which increases linearly towards the free boundaries, in contrast to the asymptotic solution of ref. [4] where an infinite system with decaying boundary conditions was considered. The constant $c$ can be determined by matching the solution (27) to the small- $r^{\prime}$ solution of ref. 44.

In $O\left(\ddot{X}_{j}\right)$ we obtain by the above methods

$$
m_{2}=p b x_{j}^{\prime} \ddot{X}_{j}
$$

with $b=c /(4 \delta)$, and

$$
\phi_{2}=\frac{q}{8 \delta} \ln r^{\prime}\left(x_{2}^{\prime} \ddot{X}_{1}-x_{1}^{\prime} \ddot{X}_{2}\right) .
$$

Here $m_{2} \sim \cos \chi^{\prime}$ and $\phi_{2} \sim \sin \chi^{\prime}$, where $\chi^{\prime}$ is the angle between $\vec{r}^{\prime}$ and $\ddot{\vec{X}}$.

The results of this section can be tested by looking at snapshots of the orientation of the spins in our computer simulations for the discrete system (the simulations are discussed in section 5). The dynamic parts of $\phi$ are very difficult to observe because $\phi_{0}$ must be substracted first. But this depends very sensitively on the vortex position, which is known only within a certain accuracy. However, $m_{0}$ vanishes exponentially for $r^{\prime} \gg r_{V}$. Here the dynamic parts of $m$ can be observed directly. Though $m_{2}$ increases linearly with $r^{\prime}$ while $m_{1}$ decays, $m_{2}$ generally cannot be distinguished clearly from $m_{1}$ because generally $|\ddot{\vec{X}}| \ll|\dot{\vec{X}}|$. In order to observe $m_{2}$ nevertheless, we have selected specific points of the vortex trajectories in section 5: At the turning points the acceleration has a maximum while the velocity is small. Here the predicted $\cos \chi^{\prime}$-dependence of $m_{2}$ and its linear increase towards the boundaries are seen very clearly (Figs. 1 and 3). In the middle between two turning points the trajectory is nearly straight and the acceleration is small. Here $m_{2}$ is in fact barely visible (Fig. 2), instead several humps can be observed which are probably produced by $m_{1}$. By the evaluation of contour plots of the above snapshots we estimate $b \approx 2.5$ for the parameter in $(28)$. This parameter is the only one we need for the calculation of the integrals in the following section.

\section{Mass, third-order gyrotensor and solution of the equation of motion}

Since we consider very slow motion we need to calculate only the rest mass, i.e. in Eq. (13) the derivations with respect to $X_{i}$ are applied only to the static parts of $\phi$ and $m$. As $\phi_{1}$ and $m_{1}$ depend linearly on the velocity,

$$
M_{i j}=\int d^{2} r\left\{\frac{\partial \phi_{0}}{\partial X_{i}} \frac{\partial m_{1}}{\partial \dot{X}_{j}}-\frac{\partial \phi_{1}}{\partial \dot{X}_{j}} \frac{\partial m_{0}}{\partial X_{i}}\right\}
$$


is the rest mass. In the same way we obtain the constant part of (14) by

$$
A_{i j}=\int d^{2} r\left\{\frac{\partial \phi_{0}}{\partial X_{i}} \frac{\partial m_{2}}{\partial \ddot{X}_{j}}-\frac{\partial \phi_{2}}{\partial \ddot{X}_{j}} \frac{\partial m_{0}}{\partial X_{i}}\right\} .
$$

An analytic calculation is possible if we choose a circular system (radius $L$ ) and consider a vortex with its center $\vec{X}$ at the circle center, which is chosen as origin. We divide the integration region into an inner part $0 \leq r \leq a_{c}$ and an outer part $a_{c} \leq r \leq L$, where we choose $a_{c} \gg r_{V}$. The inner regions yield $L$-independent contributions, while the contributions from the outer region turn out to increase with $L$ and thus dominate for large $L$. As $m_{0}$ decays exponentially according to (19), the second terms in (30) and (31) give no contribution for the outer region. Therefore we need to calculate only the first terms and obtain with (18), (26) and (28)

$$
\begin{aligned}
M_{i j}=M \delta_{i j} \quad, \quad M=\frac{\pi}{4 \delta} \ln \frac{L}{a_{c}}+\text { const. } \\
A_{i j}=A \epsilon_{i j} \quad, \quad A=\frac{G b}{4}\left(L^{2}-a_{c}^{2}\right)+\text { const. }
\end{aligned}
$$

where $\delta_{i j}$ is the 2 -d unit matrix. The constants are the above $L$-independent contributions from the inner region. They depend on $a_{c}$, but together with the other $a_{c}$-dependent terms they must add up to $a_{c}$-independent constants $M_{0}$ and $A_{0}$ because the final result

$$
M=\frac{\pi}{4 \delta} \ln L+M_{0} \quad, \quad A=\frac{G b}{4} L^{2}+A_{0}
$$

must not depend on how the integration region is divided.

If the vortex center is not at the circle center the situation is less symmetric, but $\mathbf{M}$ is still diagonal (in a system with axes parallel and orthogonal to $\vec{X}$ ) and the $A_{i i}$ are still zero. The non-vanishing matrix elements must be calculated by numerical integration. However, in our simulations the mean vortex trajectory is a circle with radius $R_{0} \ll L$ around the circle center. Therefore the differences between $M_{11}$ and $M_{22}$ and those between $A_{12}$ and $-A_{21}$ are small and will be neglected in the following.

For the solution of the equation of motion (10) we consider a small displacement from a mean trajectory $\vec{X}^{0}(t)$

$$
\vec{X}(t)=\vec{X}^{0}(t)+\vec{x}(t)
$$

We consider a situation where the force is always pointing in the $X_{1}$-direction and expand to $1^{\text {st }}$ order around $X_{1}^{0}=R_{0}$

$$
F=F_{0}+F_{0}^{\prime} x_{1}
$$

The mean trajectory is a parallel to the $X_{2}$-axis, namely $X_{1}^{0}=R_{0}, X_{2}^{0}=V_{0} t$, where $V_{0}=F_{0} / G$ is positive for a vortex with $q p=1$. 
For the displacement $\vec{x}$ we get two linear equations

$$
\begin{aligned}
A \dddot{x}_{2}+M \ddot{x}_{1}+G \dot{x}_{2} & =F_{0}^{\prime} x_{1} \\
-A \dddot{x}_{1}+M \ddot{x}_{2}-G \dot{x}_{1} & =0
\end{aligned}
$$

which are solved by

$$
\begin{aligned}
& x_{1}=a_{1} \cos \omega_{1} t+a_{2} \cos \omega_{2} t \\
& x_{2}=b_{1} \sin \omega_{1} t+b_{2} \sin \omega_{2} t
\end{aligned}
$$

with

$$
\omega_{1,2}^{2}=\frac{2 G A+M^{2}}{2 A^{2}} \mp \sqrt{\frac{\left(4 G A+M^{2}\right) M^{2}}{4 A^{4}}+\frac{M F_{0}^{\prime}}{A^{2}}} .
$$

The amplitude ratios $\kappa_{\alpha}=b_{\alpha} / a_{\alpha}$ are obtained from

$$
\begin{aligned}
\left(G-A \omega_{\alpha}^{2}\right) \omega_{\alpha} \kappa_{\alpha} & =M \omega_{\alpha}^{2}+F_{0}^{\prime} \\
G-A \omega_{\alpha}^{2} & =M \omega_{\alpha} \kappa_{\alpha}
\end{aligned}
$$

which yields

$$
\kappa_{\alpha}= \pm \sqrt{1+F_{0}^{\prime} /\left(M \omega_{\alpha}^{2}\right)}, \quad \alpha=1,2 .
$$

In order to facilitate the discussion we set $F_{0}^{\prime}=0$ (our simulations are made anyway for the case of small $F_{0}$ and $F_{0}^{\prime}$. Then we get

$$
\omega_{1,2}=\sqrt{\frac{G}{A}+\left(\frac{M}{2 A}\right)^{2}} \mp \frac{M}{2 A}
$$

with $\kappa_{1,2}= \pm 1$. As $A \sim L^{2}$ while $M \sim \ln L$ for a large system, the leading $L$ dependence of $\omega_{\alpha}$ is $1 / L$.

The frequencies $\omega_{1,2}$ form a narrow doublet. The mean frequency depends on $A$, but not on $M$

$$
\omega_{c}=\sqrt{\omega_{1} \omega_{2}}=\sqrt{G / A} \sim 1 / L .
$$

Contrary to this, the splitting of the doublet

$$
\Delta \omega=\omega_{2}-\omega_{1}=M / A \sim \frac{\ln L}{L^{2}}
$$

is proportional to the mass.

We now discuss the size dependence of the different terms in the equation of motion (10). As every time derivative contributes a factor $\omega_{\alpha} \sim 1 / L$, we see that $A \dddot{X}_{i} \sim 1 / L$ and $M \ddot{X}_{i} \sim \ln L / L^{2}$ for a large system. Therefore $A \dddot{X}_{i}$ cannot be neglected when $M \ddot{X}_{i}$ is retained. This is the reason for the discrepancies resulting from the $2^{\text {nd }}$-order equation (田), see the introduction. However, the neglection of both $A \dddot{X}_{i}$ and $M \ddot{X}_{i}$ represents a consistent approximation, namely the Thiele Eq. (四). 
We have just seen that the levels $n=1$ and $n=3$ represent consistent approximations in a hierarchy of equations of motion. Let us now include two additional time derivatives in our ansatz (\$) which yields two additional orders in the equation of motion. Using the methods of sections 3 and 4 one can show that the parameters in the $5^{\text {th }}$ - and $4^{\text {th }}$-order terms scale like $L^{4}$ and $L^{2} \ln L$, resp.. The same arguments as above show that $n=5$ is the next consistent level. Here we get 4 frequencies $\omega_{\alpha}$ (besides the trivial solution $\omega=0$ ). Neglecting for the moment the small $2^{\text {nd }}$ - and $4^{\text {th }}$-order terms, we obtain two two-fold degenerate frequencies. The inclusion of the neglected terms lifts the degeneracy and gives a spectrum of two doublets $\omega_{1,2}$ and $\omega_{3,4}$. The corresponding amplitudes $a_{\alpha}$ are free constants in a general solution which are determined by the initial conditions (only the ratios $\kappa_{\alpha}=b_{\alpha} / a_{\alpha}$ are fixed, as in (40)). It will turn out in the next section that $a_{3,4}$ are generally so small that $\omega_{3,4}$ cannot be observed. Only for very special conditions can $\omega_{3,4}$ be seen.

Finally we shortly discuss non-gyrotropic excitations which we define as having only even-order terms in the equations of motion. Examples are the kinks in the 1-d nonlinear Klein-Gordon models for which $\mathbf{G}$ and $\mathbf{A}$ vanish. Here a $4^{\text {th }}$-order equation was derived but not considered in detail [11]. Another example are the planar vortices of the 2-d anisotropic Heisenberg model (stable for $\delta>\delta_{c}$ ). Here $G=0$ in Eq. (2) because of $p=0$; all other odd-order terms also vanish because they are proportional to $p$, e.g. (31).

\section{Comparison with simulations}

A single vortex with polar coordinates $(R, \phi)$ on a circle with radius $L$ has an image vortex at $R_{i}=L^{2} / R$ and the same $\phi$ (as in 2-d electrostatics). For free boundaries the image has opposite vorticity but the same polarization $[8]$ and the force is $F(R)=$ $2 \pi /\left(R_{i}-R\right)$. The equation of motion (10) has a steady-state solution: a constant rotation on a circle $R=R_{0}, \phi(t)=\omega_{0} t$ with the following relation between $R_{0}$ and $\omega_{0}$ :

$$
\text { - } A \omega_{0}^{3} R_{0}-M \omega_{0}^{2} R_{0}+G \omega_{0} R_{0}=F\left(R_{0}\right) .
$$

This circle is identified with the mean trajectory around which we observe oscillations in the simulations (details are given in the Appendix). In Fig. th the radial coordinate $R(t)$ is plotted vs. the coordinate $R_{0} \phi(t)$ in azimuthal direction. This figure already shows qualitatively that two frequencies are involved. In fact, the Fourier spectra for $r(t)=R(t)-R_{0}$ and $\varphi(t)=\phi(t)-\omega_{0} t$ in Fig. 5 clearly show two dominant frequencies $\omega_{1,2}$. The phase shifts $\delta_{1,2}$ are approximately $\pi / 2$ and $-\pi / 2$ (Table 11). Thus the simulation data are described very accurately by

$$
\begin{aligned}
r(t) & =a_{1} \cos \omega_{1} t+a_{2} \cos \omega_{2} t \\
R_{0} \varphi(t) & =b_{1} \sin \omega_{1} t+b_{2} \sin \omega_{2} t
\end{aligned}
$$

with

$$
\operatorname{sign} \kappa_{\alpha}= \pm 1 \quad, \alpha=1,2
$$


As there are displacements not only in radial direction but also in azimuthal direction the trajectories are a superposition of two cycloids with frequencies $\omega_{1,2}$.

In order to compare with our theory we must solve the equation of motion (10) in polar coordinates. The result agrees completely with (45), (46). The formula for $\omega_{\alpha}$ is much more complicated than the cartesian result (38) due to many additional, $\omega_{0}$-dependent terms. However, $\kappa_{\alpha}$ can be written in a rather simple form by using (44)

$$
\begin{aligned}
\kappa_{\alpha}^{2} & =1+\frac{f}{\left(M+3 A \omega_{0}\right) \omega_{\alpha}^{2}} \\
f & =F_{0}^{\prime}-\frac{F_{0}}{R_{0}}=\frac{2 \pi}{L^{2}} \frac{2 \eta^{2}}{\left(1-\eta^{2}\right)^{2}}
\end{aligned}
$$

with $\eta=R_{0} / L$. For $\eta \ll 1$, which is the case in our simulations, a short calculation shows that $\kappa_{\alpha}= \pm 1$, neglecting terms of order $\eta^{2}$ and higher. In this case it turns out that the substitution

$$
\omega_{\alpha} \pm \omega_{0} \rightarrow \nu_{\alpha} \quad, \alpha=1,2
$$

transforms the complicated eigenvalue equations for $\omega_{\alpha}$ into simpler ones

$$
\begin{aligned}
\left(G-A \nu_{\alpha}^{2}\right) \nu_{\alpha} \kappa_{\alpha} & =M \nu_{\alpha}^{2}+F_{0}^{\prime} \\
\left(G-A \nu_{\alpha}^{2}\right) \nu_{\alpha} & =\left(M \nu_{\alpha}^{2}+F_{0} / R_{0}\right) \kappa_{\alpha} .
\end{aligned}
$$

Here the difference between $F_{0}^{\prime}$ and $F_{0} / R_{0}$ must be neglected to be consistent with the above neglection of $f$ in (47). We note in passing that this difference and the one between $\left|\kappa_{1}\right|$ and $\left|\kappa_{2}\right|$ are responsible for the deviations of $\mathbf{M}$ and $\mathbf{A}$ from the symmetries in (32a) and (32b), which are discussed below these equations.

As we know only the size dependence of $M$ and $A$, it does not make sense to solve (50a), (50b) for $\nu_{\alpha}$. Instead, we calculate $M$ and $A$ as functions of $\nu_{\alpha}$, or $\omega_{\alpha}$ and $\omega_{0}$, which have been measured in simulations for different system sizes:

$$
\begin{aligned}
M & =\left(B_{1} \nu_{2}^{3}-B_{2} \nu_{1}^{3}\right) / D \\
A & =\left(B_{2} \kappa_{1} \nu_{1}^{2}-B_{1} \kappa_{2} \nu_{2}^{2}\right) / D
\end{aligned}
$$

with

$$
\begin{aligned}
B_{\alpha} & =G \nu_{\alpha}-\kappa_{\alpha} F_{0} / R_{0} \\
D & =\nu_{1}^{2} \nu_{2}^{2}\left(\kappa_{1} \nu_{2}-\kappa_{2} \nu_{1}\right) .
\end{aligned}
$$

The results in Table 2 have to be compared with (33), which was calculated for $R_{0}=0$, however. Therefore we take only the data for small $R_{0} / L=\eta$, e.g. those for $\eta \approx 0.17$. The data for $A$ are well represented by $A=A_{0}+C L^{\alpha}$ with $\alpha=2.002$, $C=4.67$ and $A_{0}=40$. This agrees perfectly with the $L^{2}$-dependence in (33). From $C$ we obtain $b=2.97$, which agrees rather well with the value 2.5 which was estimated at the end of section 3. However, the values for $M$ are practically independent of 
$L$, in contrast to the logarithmic dependence in (33). This can be explained by the following: In contrast to the $1 / r^{\prime}$-decay in (26), $m_{1}$ seems to go to an $L$-dependent constant at the boundary (however, this is difficult to see because only $m_{1}+m_{2}$ is observed, and $m_{2}$ is never exactly zero). At this point we must realize that Eq. (26) is only an approximation, because for $\phi_{0}$ in (25) we have taken (18), i.e., the contribution of the image vortex has been neglected. This effect and others, such as the splitting of the $M_{i i}$-components for $R_{0} \neq 0$ and the influence of a second vortex in the system, will be considered in forthcoming work.

The last point in this paper is the observation of additional frequency doublets which can be related to higher levels of the hierarchy (see the penultimate paragraph of section 4). We have performed simulations for a vortex in the center of a square system with antiperiodic boundary conditions. In this particular case there are many image vortices at positions which can be calculated as in electrostatics. The forces from all the images cancel exactly, therefore the vortex in the center is influenced only by the small pinning forces from the lattice. We observe that the vortex center moves on small-amplitude cycloidal trajectories, which are either completely inside a lattice cell or which go over severall cells. However, the spectrum is always practically the same which means that the pinning forces do not substantially influence the frequencies. In Fig. 0 we clearly see not only a second doublet but also a third one, which we want to relate to the $5^{\text {th }}$ and $7^{\text {th }}$ levels of the hierarchy, resp. In order to show this relation we must check that the parameters $M$ and $A$ do not change dramatically when we go from the $3^{\text {rd }}$ to the $5^{\text {th }}$ level, for instance. On this level we get a $4^{\text {th }}$-order eigenvalue equation for $\omega_{\alpha}$, after splitting off the trivial solution. We need not solve this equation because we want to calculate $M, A, \ldots$ as functions of the $\omega_{\alpha}$ 's. This is achieved by using Vieta's rules which express the coefficients of an $n^{\text {th }}$-order polynominal equation by its roots. Table 3 contains the observed $\omega_{\alpha}$ 's and the resulting values for $M$ and $A$, which in fact do not differ much on the different levels of the hierarchy. We remark that this test is very sensitive: Small changes in $\omega_{\alpha}$ result in large changes in the values of $M$ and $A$.

Finally we would like to stress that the additional $\omega_{\alpha}$ 's can usually not be observed in the spectra because their amplitudes are too weak, thus our $3^{\text {rd }}$-order equation of motion is normally sufficient to describe the simulations.

\section{Discussion}

Inserting the usual travelling-wave ansatz into the Hamilton equations shows that the shape of a nonlinear coherent excitation generally depends also on its velocity $\dot{\vec{X}}$. Iterating this process we have shown that there is an additional dependence on $\ddot{\vec{X}}$,

$\dddot{\vec{X}}$, and so on. This yields a hierarchy of equations of motion for $\vec{X}(t)$. For the case of the non-planar vortices of the 2-d anisotropic Heisenberg model the odd levels of this hierarchy represent an increasingly better description of the dynamics as observed in computer simulations of the full spin system. 
Naturally the question arises why spin waves do not appear here, neither in theory nor in simulations. As to the latter point, we make two remarks: 1.) For a short time after the start of a simulation spin waves are radiated because the initial condition usually does not perfectly represent a moving vortex. In fact, if we use approximate formulas for the vortex structure, the generated spin waves are clearly observed. In our older simulations [8, 9] we eliminated them by adding a Gilbert damping term to the Landau-Lifshitz Eq. for the above short time period. However, in the present paper we use an iterative method (see Appendix) which produces a stationary vortex solution which is so good that practically no spin waves are generated. 2.) One might think that a vortex would continously radiate spin waves because it is subject to accelerations on its complicated cycloidal trajectory. Even if the amplitudes of these spin waves were too small to observe them directly, the effect should be detected indirectly by an energy loss of the vortex. Interestingly, in our simulations, even after a long time (several periods $T_{0}=2 \pi / \omega_{0}$, see (44)) there was no detectable energy loss; this suggests the possibility of additional conservation laws.

As to the theory, there is an alternative formalism [12] which includes spin waves. Instead of eqs. (8a), (8b) the following ansatz is used

$$
\begin{aligned}
\phi(\vec{r}, t) & =\phi(\vec{r}-\vec{Y}(t))+\xi(\vec{r}, t) \\
m(\vec{r}, t) & =m(\vec{r}-\vec{Y}(t))+\chi(\vec{r}, t)
\end{aligned}
$$

where the functions $\phi$ and $m$ on the r.h.s. represent the static structure of a vortex, while $\xi$ and $\chi$ represent spin waves. As the number of degrees of freedom on the r.h.s. of eqs. (53) is larger than on the l.h.s., constraints must be introduced which have the form of orthogonality relations [13, 11].

One of the constraints leads to an implicite definition of the vortex position $\vec{Y}$. This definition is global in the sense that all spins in the system are involved, in contrast to the local definition of the vortex center $\vec{X}$ that has been used in the present paper (see Appendix). Consequently different trajectories $\vec{Y}(t)$ and $\vec{X}(t)$ are obtained by analyzing the same simulation data. $\vec{Y}(t)$ turns out to be equivalent to the mean trajectory $\vec{R}_{0}(t)$ belonging to the cycloidal trajectories $\vec{X}(t)$ obtained in the present paper.

In the alternative formalism the cycloidal trajectories are obtained by a coupling of $\vec{Y}(t)$ to certain quasi-local spin wave modes. Far away from the vortex center these modes are extended while they are localized in the core region [14]. Quasilocal eigenmodes are in contrast to the truly localized, intrinsic modes of e.g. the kinks in the $\phi^{4}$-model [15]. An exact numerical diagonalization for a small system $(L=20)$ with one vortex shows that essentially only two modes are involved. Their frequencies are identical to those of the doublet which we observe in the spectrum of the cycloidal trajectories (e.g., Table 1 and Fig. 5). If higher-order doublets can be seen in the spectrum (like in Fig. 6), they can also be identified with spin waves. Hence, going e.g. from the $3^{\text {rd }}$ to the $5^{\text {th }}$ level of the hierarchy in the present paper corresponds to taking into account two additional spin wave modes in the alternative 
formalism. This strong model selection may also be responsible for inhibiting spin wave emission by the vortex motion as noted above.

\section{Acknowledgements}

Work at Los Alamos National Laboratory was sponsored by the United States Department of Energy. Travel between Los Alamos and Bayreuth was partially supported by NATO Collaborative Research Grant No. 0013/89.

\section{Appendix}

In this appendix we describe the numerical details of our work.

The time evolution of classical spin systems is governed by the Landau-Lifshitz equation

$$
\dot{\vec{S}}_{k}=\vec{S}_{k} \times \vec{H}_{k} \quad, \quad \vec{H}_{k}=-\frac{\partial H}{\partial \vec{S}_{k}}
$$

The index $k$ denotes the site-coordinates of a 2-d square lattice. The local field $\vec{H}_{k}$ is for our model (3) given by

$$
\vec{H}_{k}=J \sum_{(k l)}\left[S_{l}^{x} \vec{e}_{x}+S_{l}^{y} \vec{e}_{y}+(1-\delta) S_{l}^{z} \vec{e}_{z}\right]
$$

where here the sum runs over all the nearest neighbors of $k$ not including $k$ itself.

Equation (54) with (55) was numerically integrated using a forth-order RungeKutta scheme with a time step of 0.04 (in time units $(J S)^{-1}$ ). Either free bound-

ary conditions on a circular system, i.e. $\vec{S}_{i, j}=0$ for all $(i, j)$ with $i^{2}+j^{2}>L^{2}$, or anti-periodic boundary conditions on a square system were used. With $\phi_{i, j}=$ $\tan ^{-1} S_{i, j}^{y} / S_{i, j}^{x}$ the latter are defined in the following way:

$$
\begin{array}{rll}
\phi_{i, 0}+\phi_{i, L}=\varphi_{0} & , \quad \phi_{i, 1}+\phi_{i, L+1}=\varphi_{0} \\
\phi_{0, j}+\phi_{L, j}=\varphi_{0}+q_{i n} \pi & , \quad \phi_{1, j}+\phi_{L+1, j}=\varphi_{0}+q_{i n} \pi \\
S_{i, 0}^{z}+S_{i, L}^{z}=0 & , \quad S_{i, 1}^{z}+S_{i, L+1}^{z}=0 \\
S_{0, j}^{z}+S_{L, j}^{z}=0 & , \quad S_{1, j}^{z}+S_{L+1, j}^{z}=0 .
\end{array}
$$

$L$ denotes the number of lattice sites in $x$ - and $y$-direction, respectively (i.e. the linear dimension of the system). $q_{\text {in }}$ is the total amount of vorticity in the system ( 1 or -1 for one vortex in the system) and $\varphi_{0}$ is an arbitrary constant.

The exact structure of an one-vortex solution is, even in the static case, analytically not known. Therefore we calculated the initial conditions for our simulations numerically using the following iteration scheme:

$$
\vec{S}_{k}^{(n+1)}=\frac{\left|\vec{S}_{k}^{(n)}\right|}{\left|\vec{H}_{k}^{(n)}\right|} \vec{H}_{k}^{(n)} .
$$


Normally this method is used to calculate static solutions of the Landau-Lifshitz equation. However, iterating only a few times (a typical value is 30) a pronounced vortex structure develops which is suitable to serve as the initial spin field for the simulations.

Next we explain how we have determined the vortex position(s) during a simulation. Using the discrete variant of the contour integral $\oint d \vec{r} \nabla \phi(\vec{r})$ (which yields $2 \pi q$ if the contour surrounds the vortex and zero otherwise), only the lattice plaquette can be identified in which the vortex necessarily has to reside. We estimated the precise position according to the formulas

$$
\begin{aligned}
& X_{1}=\frac{1}{2} \frac{-\sin \left[q^{-1}\left(\phi_{1}+\phi_{2}-\phi_{3}-\phi_{4}\right)\right]}{\cos \left[q^{-1}\left(\phi_{1}+\phi_{2}-\phi_{3}-\phi_{4}\right)\right]-\sin \left[q^{-1}\left(\phi_{1}-\phi_{2}+\phi_{3}-\phi_{4}\right)\right]-\cos \left[q^{-1}\left(\phi_{1}-\phi_{2}+\phi_{3}-\phi_{4}\right)\right]} \\
& X_{2}=\frac{1}{2} \frac{\sin \left[q^{-1}\left(\phi_{1}-\phi_{2}-\phi_{3}+\phi_{4}\right)\right]}{\cos \left[q^{-1}\left(\phi_{1}-\phi_{2}-\phi_{3}+\phi_{4}\right)\right]+\sin \left[q^{-1}\left(\phi_{1}-\phi_{2}+\phi_{3}-\phi_{4}\right)\right]-\cos \left[q^{-1}\left(\phi_{1}-\phi_{2}+\phi_{3}-\phi_{4}\right)\right]}
\end{aligned}
$$

where $\phi_{1}, \ldots, \phi_{4}$ label the angles (cf. (15)a)) of the four innermost spins at the vortex core, beginning with the spin downleft and surrounding the vortex counter-clockwise. $X_{1}$ and $X_{2}$ are measured in units of the lattice constant. The underlying coordinate system has its origin at the center of the lattice plaquette under consideration, i.e. $X_{1}$ and $X_{2}$ range between $[-0.5,0.5]$ for 'reasonable' values of $\phi_{1}, \ldots, \phi_{4}$.

Our basic assumption in deriving (57) was that $\phi_{1}, \ldots, \phi_{4}$ are distributed according to the static solution (18). Considering only differences, this eliminates the rather annoying constant $\varphi_{0}$ in (18), we can derive the following four equations:

$$
\begin{aligned}
\tan \left[q^{-1}\left(\phi_{1}-\phi_{2}\right)\right] & =-\frac{1}{2} \frac{1+2 X_{2}}{X_{1}^{2}+X_{2}^{2}+X_{2}} \\
\tan \left[q^{-1}\left(\phi_{2}-\phi_{3}\right)\right] & =-\frac{1}{2} \frac{1-2 X_{1}}{X_{1}^{2}+X_{2}^{2}-X_{1}} \\
\tan \left[q^{-1}\left(\phi_{3}-\phi_{4}\right)\right] & =-\frac{1}{2} \frac{1-2 X_{2}}{X_{1}^{2}+X_{2}^{2}-X_{2}} \\
\tan \left[q^{-1}\left(\phi_{4}-\phi_{1}\right)\right] & =-\frac{1}{2} \frac{1+2 X_{1}}{X_{1}^{2}+X_{2}^{2}+X_{1}} .
\end{aligned}
$$

We formally assume that the quantities $X_{1}, X_{2}$ and $X_{1}^{2}+X_{2}^{2}$ are independent of each other. Eqs. $(58 \mathrm{~b}, \mathrm{~d})$ then constitute a linear system in $X_{1}$ and $X_{1}^{2}+X_{2}^{2}$ and analogeously (58/a,c) in $X_{2}$ and $X_{1}^{2}+X_{2}^{2}$. Both set of equations can easily be solved and yield after some further trigonometric steps the above formulas for $X_{1}$ and $X_{2}$.

Of course in general, $\phi_{1}, \ldots, \phi_{4}$ are not distributed according to the $\tan ^{-1}$-function. Although then (58a-d) do (most probably) not have a solution at all, the Eqs. (57) can still be justified in the sense that they provide an approximation of the minimal residual of $(58 \mathrm{a}-\mathrm{d})$. Anyway, the reasonability of (57) is also acknowledged by the numerical simulations which show that trajectories are smoothly connected when the vortex leaves one lattice plaquette and enters another one.

\section{References}


[1] A. A. Thiele, Phys. Rev. Lett. 30, 230 (1973).

[2] A. A. Thiele, J. App. Phys. 45, 377 (1974).

[3] D. L. Huber, Phys. Rev. B26, 3758 (1982).

[4] M. E. Gouvea, G. M. Wysin, A. R. Bishop, and F. G. Mertens, Phys. Rev. B39, 11840 (1989).

[5] G. M. Wysin and A. R. Völkel, Phys. Rev. B49, 8780 (1994).

[6] G. M. Wysin, F. G. Mertens, A. R. Völkel, and A. R. Bishop, in Nonlinear Coherent Structures in Physics and Biology, edited by K. H. Spatschek and F. G. Mertens, Plenum Press (1994).

[7] M. E. Gouvea, F. G. Mertens, A. R. Bishop, and G. M. Wysin, J. Phys.: Condens. Matter 2, 1853 (1990).

[8] F. G. Mertens, G. M. Wysin, A. R. Völkel, A. R. Bishop, and H. J. Schnitzer, in Nonlinear Coherent Structures in Physics and Biology, edited by K. H. Spatschek and F. G. Mertens, Plenum Press (1994).

[9] A. R. Völkel, G. M. Wysin, F. G. Mertens, A. R. Bishop, and H. J. Schnitzer, Phys. Rev. B50, 12711 (1994).

[10] F. G. Mertens, H. J. Schnitzer, and A. R. Bishop, conference on Nonlinear Coherent Structures in Physics and Biology, Edinburgh 1995, unpublished.

[11] R. Boesch, P. Stancioff, and C. R. Willis, Phys. Rev. B38, 6713 (1988).

[12] H. J. Schnitzer, Ph. D. thesis (in German), University of Bayreuth (1996); H. J. Schnitzer, F. G. Mertens, and A. R. Bishop, to be published.

[13] E. Tomboulis, Phys. Rev. D12, 1678 (1975).

[14] G. M. Wysin and A. R. Völkel, Phys. Rev. B52, 7412 (1995).

[15] A. R. Bishop, J. A. Krumhansl, and S. E. Trullinger, Physica 1D, 1 (1980). 


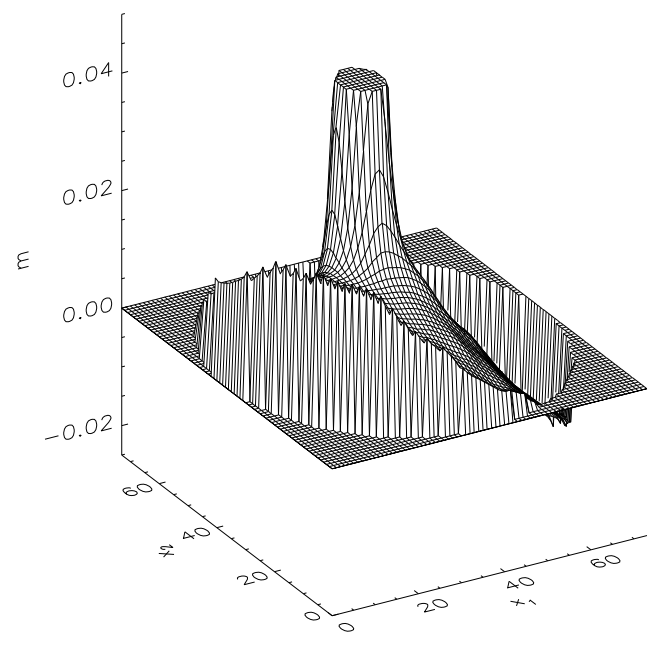

Figure 1: Out-of-plane structure of the vortex at the $7^{\text {th }}$ turning point of the trajectory in Fig. 4 . Here the acceleration has a maximum and points in radial direction, while the velocity is small and points in azimuthal direction.

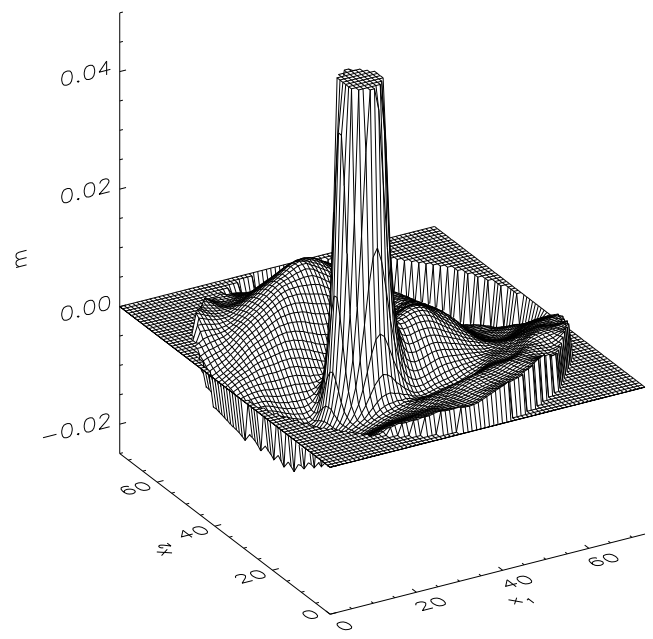

Figure 2: Out-of-plane structure at the middle between the $7^{\text {th }}$ and the $8^{\text {th }}$ turning point of Fig. 4 . Here the velocity has a maximun and points in radial direction, while the acceleration has a minimum. 


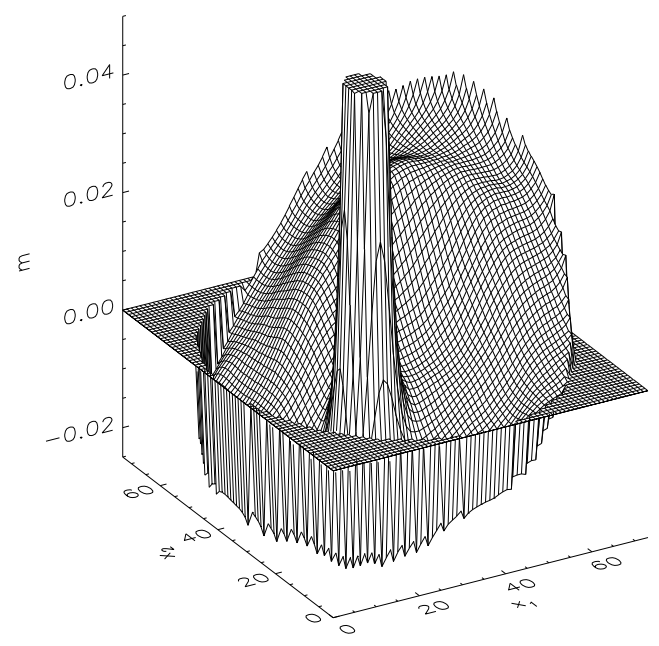

Figure 3: Same as in Fig. 1, but at the $8^{\text {th }}$ turning point, where the accelaration points in negativ radial direction.

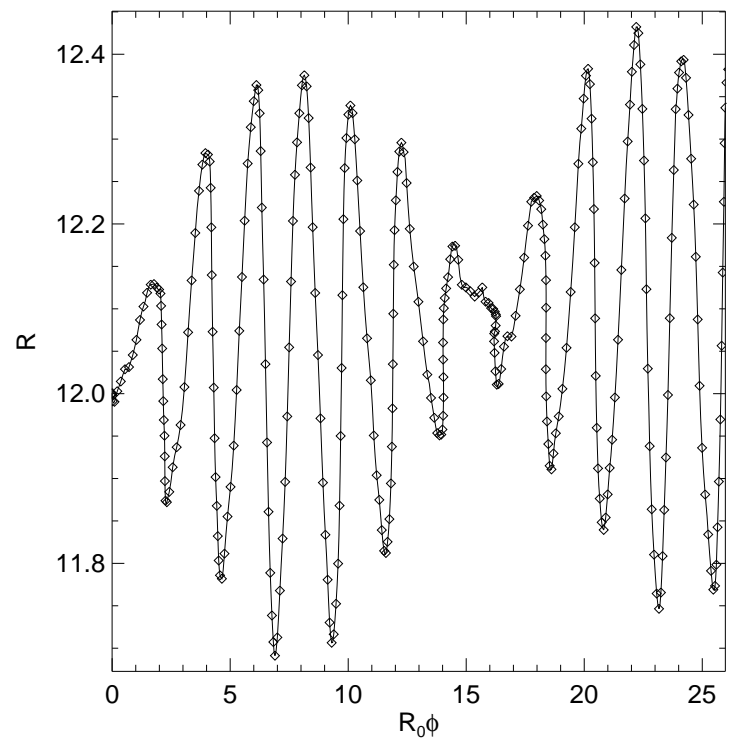

Figure 4: First part of the trajectory of a vortex with $q=p=1$ on a circular system with radius $L=36$ and free boundary conditions. The small diamonds $(\diamond)$ mark the position of the vortex in time intervals of $10(\mathrm{JS})^{-1}$. 

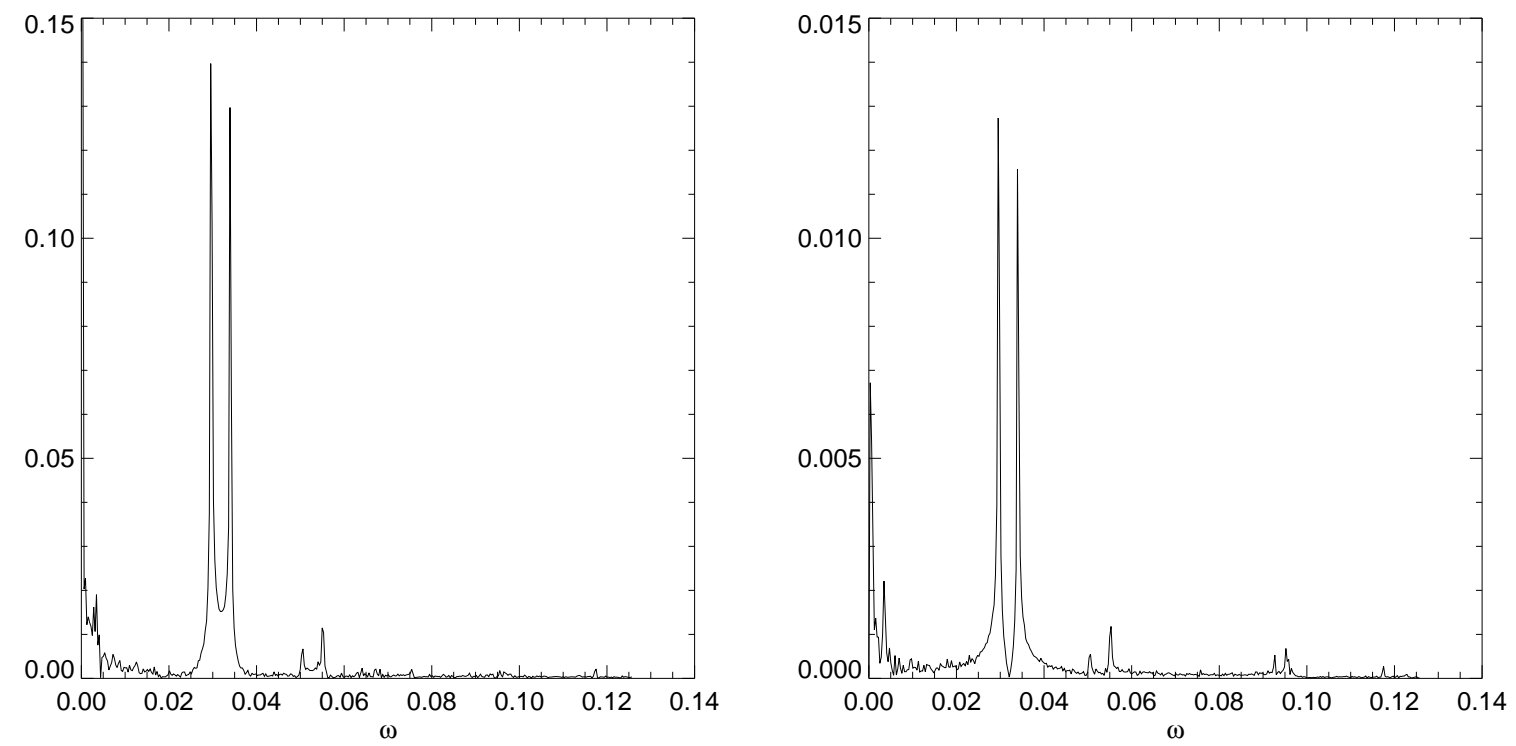

Figure 5: (a) Fourier spectrum of the radial displacements $r(t)=R(t)-R_{0}$, from simulation data for $0 \leq t \leq 20000(J S)^{-1}$. The first part of the trajectory is plottet in Fig. 因. (b) Spectrum of the aximuthal displacements $\varphi(t)=\phi(t)-\omega_{0} t$.

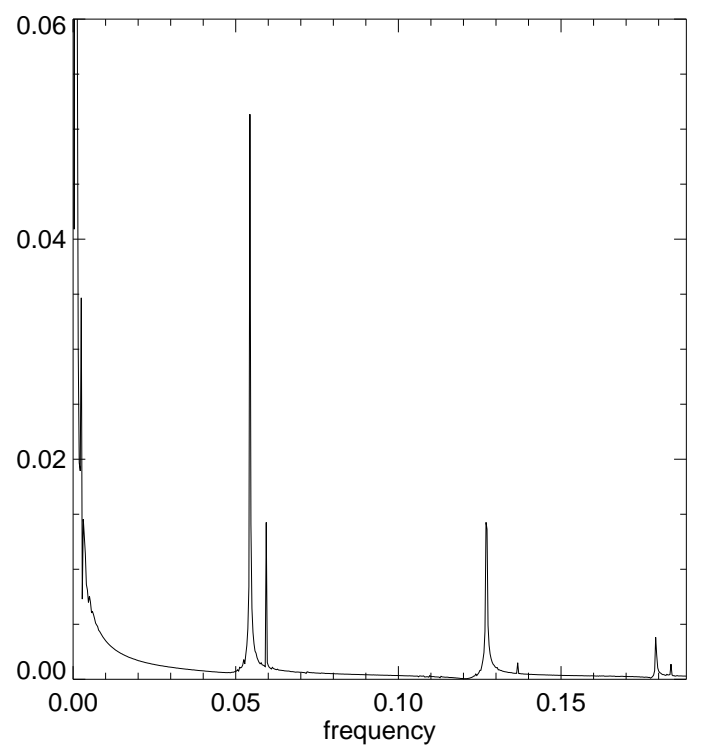

Figure 6: Spectrum of one of the cartesian coordinates of a vortex on a $50 \times 50$ square lattice with antiperiodic boundary conditions, from simulation data for $0 \leq$ $t \leq 20000(J S)^{-1}$. 
Table 1: Observed data from the trajectory of a vortex with $q=p=1$ on a circle of radius $L$.

\begin{tabular}{ccccccccc}
$L$ & $R_{0}$ & $\omega_{0} / 10^{-3}$ & $\omega_{1} / 10^{-2}$ & $\omega_{2} / 10^{-2}$ & $\delta_{1}$ & $\delta_{2}$ & $\kappa_{1}$ & $\kappa_{2}$ \\
\hline 24 & 4.03 & 1.84 & 4.257 & 5.302 & 1.57 & -1.57 & 1.01 & -1.06 \\
24 & 6.02 & 1.90 & 4.257 & 5.276 & 1.56 & -1.56 & 1.03 & -1.11 \\
24 & 8.02 & 2.01 & 4.261 & 5.249 & 1.56 & -1.56 & 1.06 & -1.18 \\
\hline 36 & 5.80 & 0.814 & 2.958 & 3.426 & 1.57 & -1.57 & 1.00 & -1.02 \\
36 & 8.28 & 0.823 & 2.962 & 3.417 & 1.52 & -1.50 & 1.02 & -1.09 \\
36 & 9.87 & 0.852 & 2.964 & 3.412 & 1.56 & -1.56 & 1.06 & -1.12 \\
36 & 12.25 & 0.889 & 2.966 & 3.404 & 1.56 & -1.57 & 1.05 & -1.14 \\
\hline 72 & 16.11 & 0.205 & 1.546 & 1.661 & 1.43 & -1.42 & - & - \\
72 & 24.20 & 0.218 & 1.548 & 1.657 & 1.39 & -1.40 & 1.12 & -1.22 \\
72 & 31.95 & 0.243 & 1.550 & 1.653 & 1.45 & -1.46 & 1.24 & -1.37
\end{tabular}

Table 2: Parameters $M$ and $A$ of the equation of motion, calculated from (51a), (51b). Values in parentheses are extrapolated.

\begin{tabular}{ccccc}
$L$ & $R_{0}$ & $\eta=R_{0} / L$ & $M$ & $A$ \\
\hline 24 & 4.03 & 0.168 & 13.7 & 2750 \\
24 & 6.02 & 0.251 & 12.5 & 2764 \\
24 & 8.02 & 0.334 & 10.8 & 2776 \\
\hline 36 & 5.80 & 0.161 & 13.9 & 6167 \\
36 & 8.28 & 0.230 & 12.9 & 6174 \\
36 & 9.87 & 0.274 & 12.0 & 6180 \\
36 & 12.25 & 0.340 & 10.7 & 6190 \\
\hline 72 & 12.0 & 0.167 & $(13.8)$ & $(24416)$ \\
72 & 16.1 & 0.224 & 13.0 & 24436 \\
72 & 24.2 & 0.336 & 10.7 & 24477 \\
72 & 31.9 & 0.443 & 7.4 & 24504
\end{tabular}


Table 3: Parameters $M$ and $A$ of the third-order equation of motion, compared to the parameters $M, A, B, C$ of the fifth-order equation, where $B$ and $C$ belong to $\vec{X}^{(4)}$ and $\vec{X}^{(5)}$, respectively. The $\omega_{\alpha}$ 's are observed in vortex motion on $2 L \times 2 L$ square lattices with antiperiodic boundary conditions.

\begin{tabular}{ccc}
$L$ & 10 & 25 \\
\hline$\omega_{1} / 10^{-1}$ & 1.376 & 0.5435 \\
$\omega_{2} / 10^{-1}$ & 1.696 & 0.5938 \\
$\omega_{3} / 10^{-1}$ & 3.749 & 1.2692 \\
$\omega_{4} / 10^{-1}$ & 4.362 & 1.3670 \\
\hline$M\left(3^{\text {rd }}\right)$ & 8.62 & 9.79 \\
$A\left(3^{\text {rd }}\right)$ & 269 & 1947 \\
\hline$M\left(5^{\text {th }}\right)$ & 10.98 & 13.42 \\
$A\left(5^{\text {th }}\right)$ & 304 & 2300 \\
$B\left(5^{\text {th }}\right)$ & 153 & 1689 \\
$C\left(5^{\text {th }}\right)$ & 1646 & 111991
\end{tabular}

